\title{
Progress in the Independent Certification of Mizar Mathematical Library in Isabelle
}

\author{
Cezary Kaliszyk \\ Universität Innsbruck, \\ Technikerstr. 21a/2, 6020 Innsbruck, Austria \\ Email: cezary.kaliszyk@uibk.ac.at
}

\author{
Karol Pąk \\ University of Białystok, \\ Ciołkowskiego 1M, 15-245 Białystok, Poland \\ Email: pakkarol@uwb.edu.pl
}

\begin{abstract}
The Mizar Mathematical Library is one of the largest collections of machine understandable formal proofs encompassing many areas of today mathematics including results from algebra, analysis, topology, and lattice theory. The Mizar system has so far been the only tool able to completely process, certify, and make use of these developments. In this paper, we present the progress in the development of an independent certification mechanism of Mizar proofs based on the Isabelle logical framework. The approach allows rechecking the Mizar formal proofs based on a more succinct and more precisely specified formal infrastructure. Additionally, it necessitates a full formal specification of the mechanisms that ensure the correctness of the defined objects, in particular, the proofs that such mechanisms are correct. The development already covers an important part of the Mizar library foundations. We improve the mechanism for defining Mizar structures and show that it permits simpler validation of proof developments involving such objects. To demonstrate this, we perform a complete translation of the Mizar net of basic algebraic structures including their attributes and certify all the corresponding proofs in Isabelle.
\end{abstract}

\section{INTRODUCTION}

C OMPUTER certified formal proofs are today one of the most important techniques used in formal methods. They are used to guarantee the correctness of compilers [1], operating systems [2], hardware [3], as well as to certify mathematical results that involve computation [4]. The Mizar system [5] is one of the oldest computer systems used to certify proofs. Its library, the Mizar Mathematical Library [6] (MML) contains today more than 1200 articles and 60000 proved theorems mainly about mathematics. The Mizar system has so far been the only tool able to process, fully certify, and make use of these formal proof developments.

Algebraic structures are one of the basic building blocks of formal proofs. They are crucial both for the foundations of mathematics and of computer science. This can be witnessed by the formal proof libraries of various interactive proof systems. Indeed, the standard library of Isabelle/HOL [7] defines more than three hundred type classes used in most of its Archive of Formal Proofs [8]. Coq uses its records which include properties in its algebraic foundations, both in the standard library [9], its constructive repository [10], and in the small scale reflection libraries [11] used as a foundation for the Four-Color theorem and the Odd-Order

Supported by Polish National Science Center grant decision $\mathrm{n}^{\circ} \mathrm{DEC}$ 2015/19/D/ST6/01473. theorem proofs. Finally, the MML [6] includes more than a hundred structures which together with different attributes correspond to thousands of different algebraic structures. $74 \%$ of the Mizar articles depend directly or indirectly on algebraic structures, including the most important domains of mathematics developed in MML, such as topological spaces, vector spaces, lattices, and fuzzy sets [12].

In this paper, we discuss the progress in our project attempting to certify the MML independently. We make use of the Isabelle logical framework [13] to specify the foundations of Mizar [14]. We further define a number of mechanisms that help to translate the Mizar definitions and proofs [15]. We investigate the set theoretic representation of algebraic structures and certify them in the Isabelle logical framework object logic corresponding to the Mizar foundations as well as translate a significant part of the Mizar algebraic structure foundations. After shortly introducing logical frameworks and Isabelle (Section II), as well as Mizar and the corresponding object logic (Section III), the particular contributions of this paper are:

- We provide an infrastructure for more elegant proofs of Mizar structure correctness conditions including structures with multiple fields (Section IV);

- We formalize all basic algebraic Mizar structures in Isabelle/Mizar together with their defining properties including structures that include other structures as components, such as a structure over a field and show that the defined Mizar structures are correctly handled in the presence of attributes and in particular that proofs about such defined algebraic structures can be concise and elegant (Section V).

\section{LOGICAL FRAMEWORKS AND ISABELLE}

Nearly all interactive proof assistants today rely on one fixed logic. This allows optimizing a system for that foundations. However, a number of systems focused on modeling actual logical systems. These are referred to as logical frameworks, and later a number of such systems became useful not only for modeling the logic but also to work in the specified logic, referred to as object logic. The three major logical frameworks are Isabelle [7], Twelf [16], and MMT [17].

Isabelle is today one of the proof systems with the largest libraries of formally proved theorems. It is based on a simple 
type theory with a shallow polymorphism that is implemented in a manually checked kernel. The meta logic provides the user with a set of primitives that makes it convenient to define object logics. The most developed object logics are higherorder logic, untyped set theory, and Lamport's temporal logic of actions.

An Isabelle formalization consists of one or more theory files. A theory is a collection of definitions, proved theorems, and notations that allow nicer presentation of terms. An Isabelle definition introduces a new identifier that is equal or equivalent (equal as a boolean predicate) to a definition body. A theorem or lemma consists of the statement and the proof. For most of the proved theorems presented in the rest of the paper, we will omit the proofs, they are fully included in the development. Each abbreviation allows for convenient input or output syntax for more complicated terms, without introducing new definitions. These are useful if such a definition would always need to be unfolded and is nicer presented as folded to the user. Most Isabelle proofs are today written in the declarative Isar style [18]. There, intermediate statements are introduced using the have keyword and justified using proof methods. For the rest of the paper, the methods and tactics used for the justifications are not essential, it is important to note their correspondence to proof steps that are considered obvious for humans. Finally the assume keyword introduces assumptions in proof blocks and show is used to denote the goal that is local to the proof block that is to be checked by Isabelle.

\section{Mizar And CORRESPONDING OBJect LogiC}

Mizar is one of the pioneering systems for mathematics formalization that is widely-used and still under active development. The Mizar project from its beginning aimed to make a system for human readable formalization of mathematics, where:

- the proof style was designed to imitate style occurring in the informal mathematical practice,

- the type system tries to express how mathematicians use mathematical objects and how they categorize them.

Therefore, Mizar uses a rich type system and proof style, which makes formalization of mathematics more intuitive and human-readable than in other systems [19], where the main idea of proofs is easy to observe [20]. Such situation occurs especially if the author of a formal proof puts additional effort to manually improve readability or uses dedicated tools [21] that optimize the NP-complete problems of improving legibility [22]. Therefore, it is not surprising that the solutions used in Mizar have been an inspiration to implement the analogical solutions in other systems.

One of these pioneering works in this field was made by J. Harrison [23] who explored the Mizar language. The result of this work was the environment Mizar Mode for HOL enabling writing proofs in a Mizar declarative way [24]. The similar solutions were implemented in other procedural proof assistants, e.g., Declare [25], Isar language for Isabelle [18], Mizar-light for HOL Light [26], miz3 for HOL Light [27],
MMode for Coq or declarative proof language (DPL) for Coq [28]. However, the similarity between these environments and Mizar system generally is limited to a few rules that are similar to the rules of the S. Jaśkowski natural deduction style [29], responsible for the universal quantifier introduction, the thesis indication, the implication elimination, the introduction of the reasoning by cases. It is worth emphasizing that the way of justification of the reasoning steps in these environments is based on tactics of the particular system that are very different from Mizar by (its equivalent can be found only in Mizar Mode for HOL [23]).

Another significant advantage of the project Mizar, from the point of view of other formal systems, is the library of mathematical knowledge formalized in the Mizar system, MML. However, the exploration of these data requires the sophisticated language constructions and types of Mizar that do not have close equivalents in other systems.

The largest translation of Mizar has been done by Urban [30] to the TPTP first-order language. Although this translation has covered the important part of the MML, it does not constitute the accurate representation of the Fraenkel operator and scheme [29]. Additional work on this solution has enabled the creation of the extensive theorems database of Mizar Problems for Theorem Proving (MPTP) that is used in the process of comparing the performance of leading systems of automatic theorem proving, as well as during the machine learning of the MizAR proof advice system [31].

Kunčar [32] has attempted to recover the Mizar system in the type system of HOL Light. This approach has enabled the translation of the first few simpler theories as transparent higher-order logic theories, however it is not applicable to the whole MML where the more advanced features of the Mizar system type are used. Difficult to reconstruct, are Mizar type mechanisms that check whether some type is a subtype of another type, generate the type of term base on types of subterm, which eliminate inconsistent instantiations and in consequence speed up the verification process. Additionally, two equal terms in Mizar can possess two incompatible types (e.g., see reconsider [33]).

The statements of the theorems in the whole MML have been exported to the MMT logical framework [34]. This allows the use of various MMT services for MML, such as searching the library or providing proof advice, however does not include an independent verification of the proofs or proof automation.

Isabelle already has an object logic Isabelle/ZF [35] based on set theory. Already the foundational axioms of ZF differ from those of Tarski-Grothendieck, and the type system introduced by Mizar is very different from any of the existing object logics in Isabelle. Furthermore, the library of Isabelle/ZF and the automation provided is quite different from that of the proposed research.

We defined an object logic that provides Mizar-like foundations in [14]. Here, we briefly remind its construction. As the foundations of Mizar are based on Jaśkowski first-order natural deduction, we start with the Isabelle/FOL object logic. We introduce one meta-level type for Mizar sets and one for 
Mizar types. We introduce the constants that correspond to sets being of particular types and to combine types (the Mizar soft type system allows intersection types [36]), the indefinite description operator, as well as the axioms that specify these constants. With the Isabelle syntax mechanisms, we allow defining Mizar like syntax for statements and definitions, which can later be used to specify the Tarski-Grothendieck foundations of set theory and translate the first few articles of the MML.

\section{Structure REQuirements}

Formalizations of computer systems often need to refer to mathematical structures. In informal computer science practice, such proofs typically use ordered tuples for such structures. For example $\langle G,+, 0\rangle$ could be an additive group and $\langle G, \cdot, 1\rangle$ a multiplicative one. In the informal approach, the expression "the group $\langle G,+, 0\rangle$ " provides two kinds of information simultaneously: a signature and its properties. The signature says that it is a structure containing the set $G$, a binary operation + and a given element of the set 0 . The properties are given as three group axioms. A formal approach to reason about such structures taken by the Mizar system attempts to avoid independent definitions of variants of structures (such as semi-group, monoid, or abelian groups) by specifying the signature separately from the adjectives that correspond to the properties of the structure.

\section{A. Structure Element Interpretation}

Every Mizar structure signature called structured type is defined as a set of assignments. Each assignment is of the form sel $\rightarrow$ spec, where sel is a unique structure element label (called selector in the Mizar language) and spec is the specification of the type of the respective element of the structure. The signature of a group is the addLoopstr structure. It is specified in MML as follows:

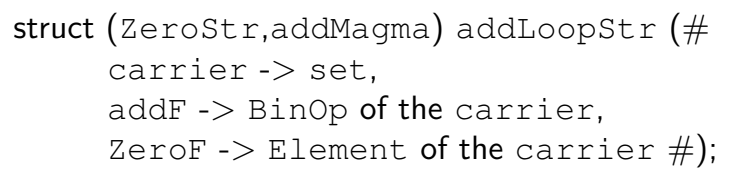

where for example addF - > Binop of the carrier denotes that + is a binary operation on the field carrier. The list of structures given in parentheses immediately after the struct keyword, namely ZeroStr, addMagma are the names of previously defined structures which contain the element 0 (ZeroStr) and a set with the binary operator (addMagma) respectively.

An Isabelle formalization of a structure type gives rise to a structure prototype. Each instance of the prototype will be a partial function, with the value corresponding to the selector having the respective type specified in the structure prototype. Definitions of this kind, even if common in informal practice, contain a recursive call. The specification can refer to other parts of the structure (in the above example addF in addLoopStr is a binary operation of the carrier). To specify this in Isabelle we further need a meta-level function which for a given object of structured type and a selector as arguments returns the term present in the object:

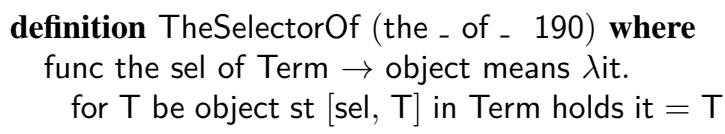

In order to use such functions in the context of structures, the actual specifications cannot be simply types, but rather functions that for a given object of a structured type as an argument returns the type. In particular, the addF element specification needs to be defined as $\lambda S$. BinOp-of the carrier of $S$. To achieve a more Mizar like formulation addF -> BinOP-of' the' carrier we further introduce abbreviations for the types with arguments:

abbreviation TheS (the" ${ }^{\prime}$ ) where

TheS $\equiv \lambda$ selector Term. the selector of Term

abbreviation BinOp_of (BinOp-of" ${ }^{\prime}$ ) where

BinOp-of' $X \equiv \lambda$ it. BinOp-of $X($ it $)$

This allows representing all assignments of the form selector $\rightarrow$ specification as a unary predicate (corresponding to the Isabelle definitions of attributes) which describes all partial functions that are the instances of the structure prototype. To allow the computation of the selector of it we add the condition that the selector is in its domain.

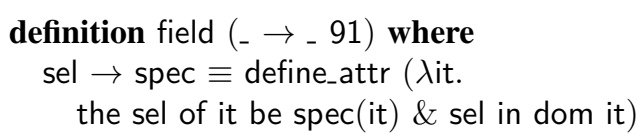

We can finally define actual structure prototypes. A new structure prototype in Isabelle corresponds to a Mizar mode (non-empty type) which is a partial function that satisfies all the constraints specified in the fields:

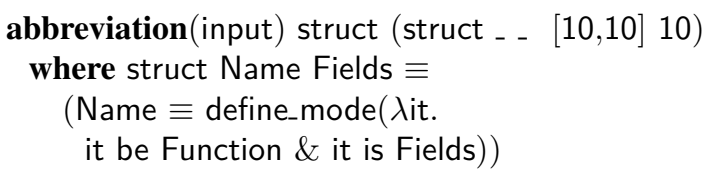

The original addLoopst $r$ can now be fully formally specified, using a syntax that is very similar to the Mizar original, while at the same time allowing a complete certification:

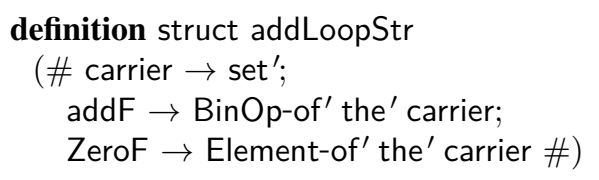

\section{B. Non-emptiness of Structure Types}

A definition of a structure prototype in Mizar provides not only the information about the types of the elements described by the signature but also ensures that there is at least one element of the structure type. For this, the Mizar checker verifies that all the defined structure specifications are non-empty. In Isabelle, we need to actually give a formal proof that the structure exists. We can achieve this by using the Hilbert choice operator $\epsilon$, providing for each assignment of the form selector $\rightarrow$ specification the pair $\langle$ selector, $\epsilon$ (specification $)\rangle$. In case of the considered addLoopstr structure prototype, we can use: 
term $\{[$ carrier, the set $]\} \cup$

$\{[$ addF, the BinOp-of the set $]\} \cup$

$\{[$ ZeroF, the Element-of the set $]\}$

Proofs of non-emptiness require a lot of effort especially with structures with more elements (some structures have as much as 12 elements). Such proofs ignore the non-emptiness proofs from the structure ancestors. We will, therefore, propose in the next Subsection IV-C a mechanism able to extend an object by the missing elements possibly changing their order. This is desired because a structure definition also implicitly defines:

- the attribute strict which means that the domain of the object contains precisely the selectors indicated in the definition and no other selectors;

- the restriction operation which restricts an object to its strict domain.

Therefore, we provide a scheme for defining the correctness of structures. We present this lemma as well as the majority of lemmas in the paper without proofs which can be found in the development.

lemma struct_scheme:

assumes $\mathrm{df}$ :

$\mathrm{S} \equiv$ define_mode $(\lambda$ it. it be Function $\&$ it is Fields)

and ex:

ex $X$ be Function st $X$ is Fields \& dom $X=D$

and monotone: for $X 1$ be Function st $X 1$ is Fields holds $\mathrm{D} \subseteq$ dom $\mathrm{X} 1$

and restriction: for $\mathrm{X} 1$ be Function st $\mathrm{X} 1$ is Fields holds $X 1 \mid D$ is Fields

shows $(x$ be $S$ iff $(x$ be Function $\& x$ is Fields $)) \&$

Ex $(\lambda x . x$ be $S) \&$ domain_of $S=D \&$

(for $X$ be $S$ holds

the_restriction_of $X$ to $S$ be (strict $S$ ) $\| S$ )

which given the subproofs for the existence condition ex and monotonicity monotone allows showing the correctness of the domain_of definition (i.e. existance and uniqueness) for the defined structure $S$, additionally deriving the equality domain_of $S=D$, where

definition domain_of (domain'_of _ 200) where

func domain_of $\mathrm{M} \rightarrow$ set means

( $\lambda$ it. (ex $X$ be $M$ st it $=$ dom $X) \&$

(for $X$ be $M$ holds it $\subseteq$ dom $X$ ))

Furthermore, by proving the restriction definition to the equality, we get the information that X | domain_of $S$ is of the structured type of $S$ which has the attribute strict, if $\mathrm{X}$ is of the structured type of $\mathrm{S}$, which completes the definition

definition restriction (the'_restriction'_of _ to _ 190)

where

func the_restriction_of $X$ to Struct $\rightarrow$ strict Struct || Struct equals

$X \mid$ domain_of Struct

where the definition of strict is as follows

definition strict :: Mode $\Rightarrow$ Attr (strict _ 200) where attr strict $M$ means

$(\lambda X . X$ be $M \&$ dom $X=$ domain_of $M)$

\section{Recursive Structure Correctness Conditions}

As discussed in the previous section, the struct_scheme lemma assumptions can be used to show the non-emptiness of a defined structure type S. However, the assumptions about each ancestor $A$ of the structure are insufficient to be usable as part of the proof for $S$. In particular, there is no condition that would correspond to restriction, which could give the information which extensions of $A$ (the extensions of the function that describe the object instance) satisfy all the assignments of A. For this reason, we propose a version of the assumption in struct_scheme with the additional fourth correctness condition

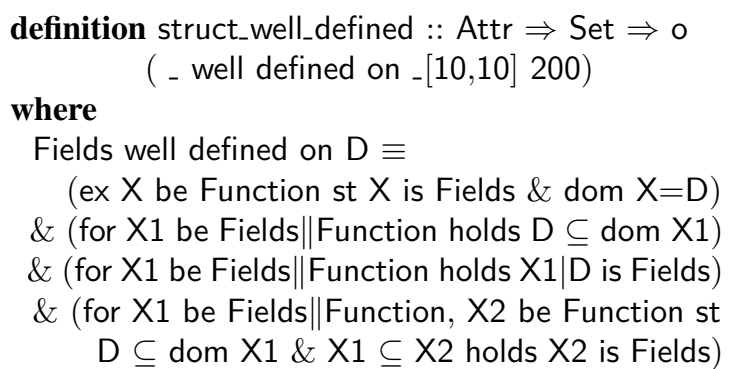

This allows a weaker defining lemma assumption

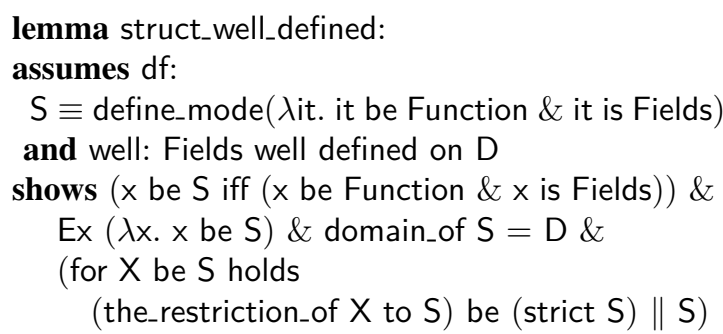

With these modifications, we can show that an existing list of assignments specified for the domain $\mathrm{D}$ can be modified by adding a new selector $\rightarrow$ specification pair assuming that the selector is not present in D so far, and the specification uses the selectors of D. An example lemma that allows extending a structure is:

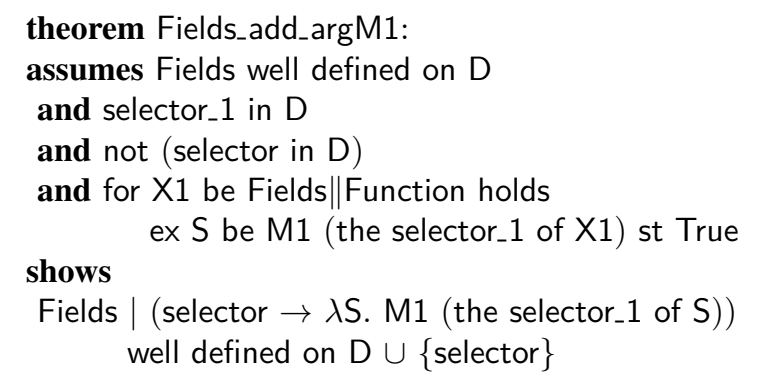

This can now be practically used to simplify the nonemptiness proof of addLoopstr using the previous proof of the well-definedness of addMagma over the set $\{$ carrier $\} \cup$ $\{$ addF $\}$ as follows:

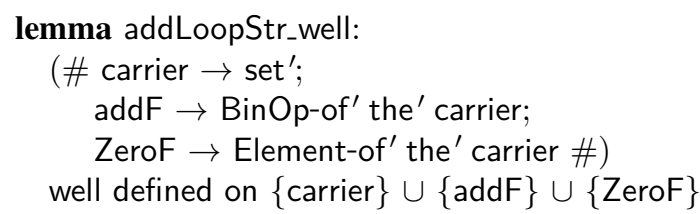




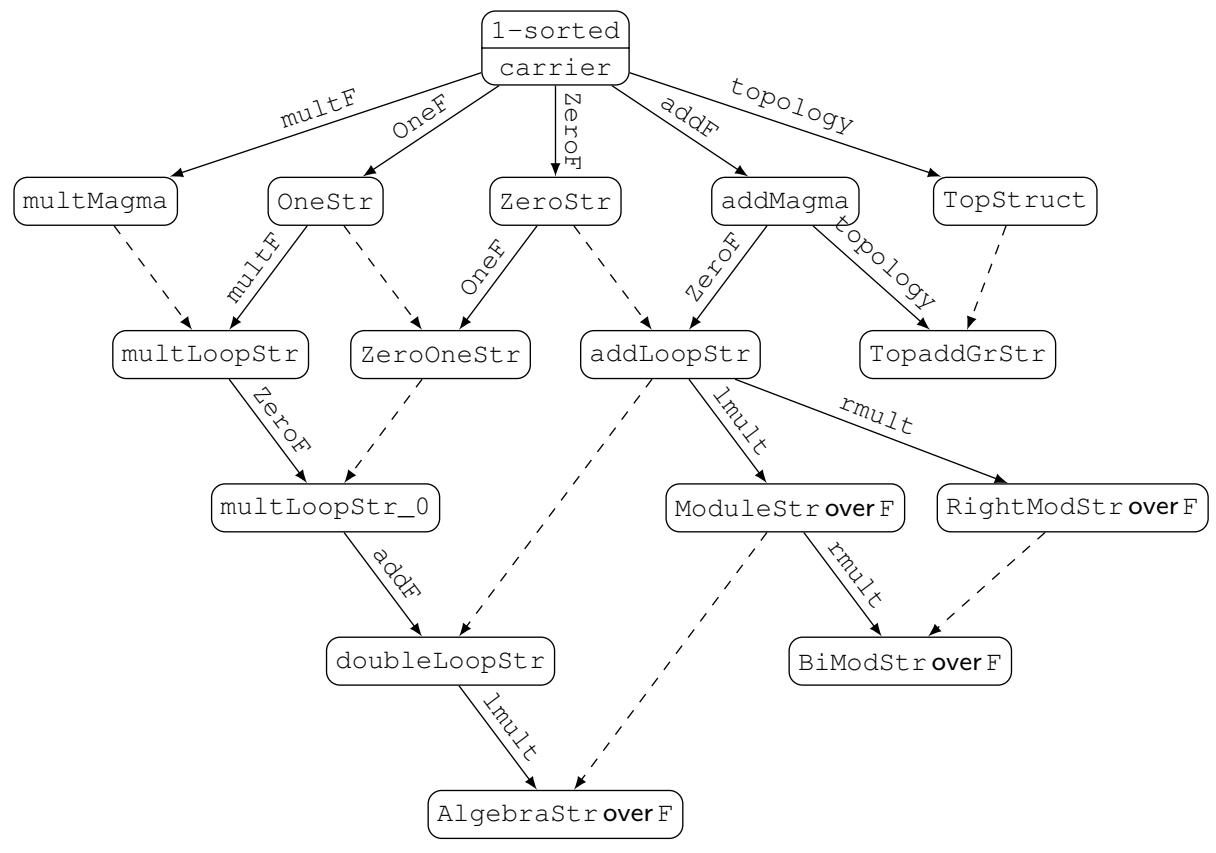

Figure 1. Net of the basic algebraic structures in the Mizar Mathematical Library following [37]. The presented ones have already been covered in our formalization. The arrow captions indicate the added selectors. Solid lines indicate the use of the ancestor structure in the well-definedness proofs, and dashed lines indicate that the ancestor structure is omitted in the proofs.

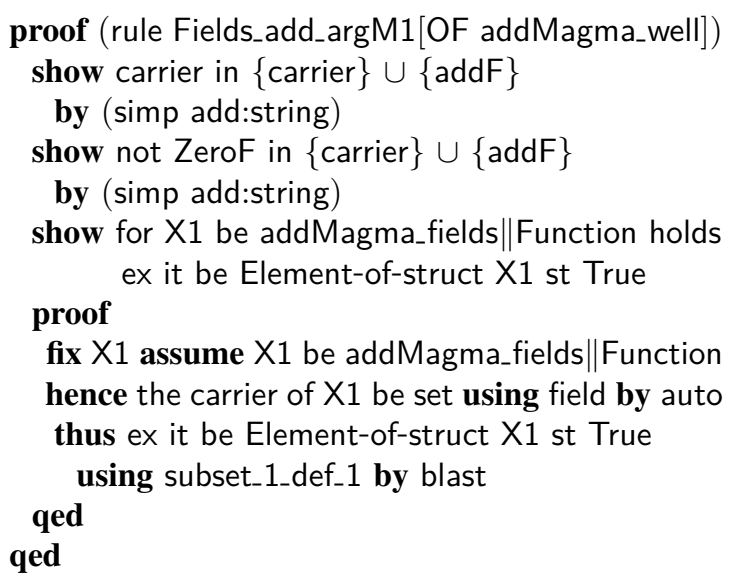

where the proof only needs to use the non-emptiness of the type Element of set. Furthermore, the fact that the carrier is a member of $\{$ carrier $\} \cup\{$ addF $\}$, as well as the fact that ZeroF is not a member of $\{$ carrier $\} \cup\{$ addF $\}$ can both be handled completely automatically by the simplifier in all such proofs.

The well-definedness of addLoopStr does not need to rely on that of the addMagma ancestor. One could instead extend the list of assignments of Zerostr by addF $\rightarrow$ Binop-of' the' carrier and change the order. For this purpose we provide the lemma:

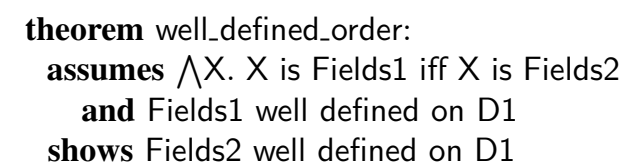

The components described above are sufficient to define all the MML structures (the basic ones are presented in Fig. 1). The construction follows the recursive element addition approach.

Even if the addLoopstr proof refers to its ancestors, the inheritance information is not provided again by struct SchemeWell. The Mizar system allows indicating this information directly in the structure definition by giving a list of all ancestors. In our approach, it is possible to prove a structure inheritance. Such proofs can be always automatically performed by the simplifier.

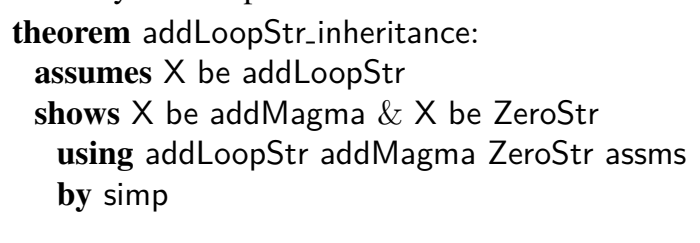

\section{Net of Basic Algebraic Structures}

Mizar structures together with the inheritance mechanisms significantly facilitate the formalization of computer systems and various domains of mathematics, as well as combining them. For this reason, structures are a challenge for our project, especially the struct_0 article which defines the elementary structures and their operations.

MML contains today 168 structure signatures. Structure signatures form a net because of multiple inheritances. Nevertheless, 135 of the signatures inherit from 1-sorted, namely the signature of structures that contain a carrier which includes some examples in most developed domains of mathematics in the MML, such as algebra, topology, and the theory of lattices. 


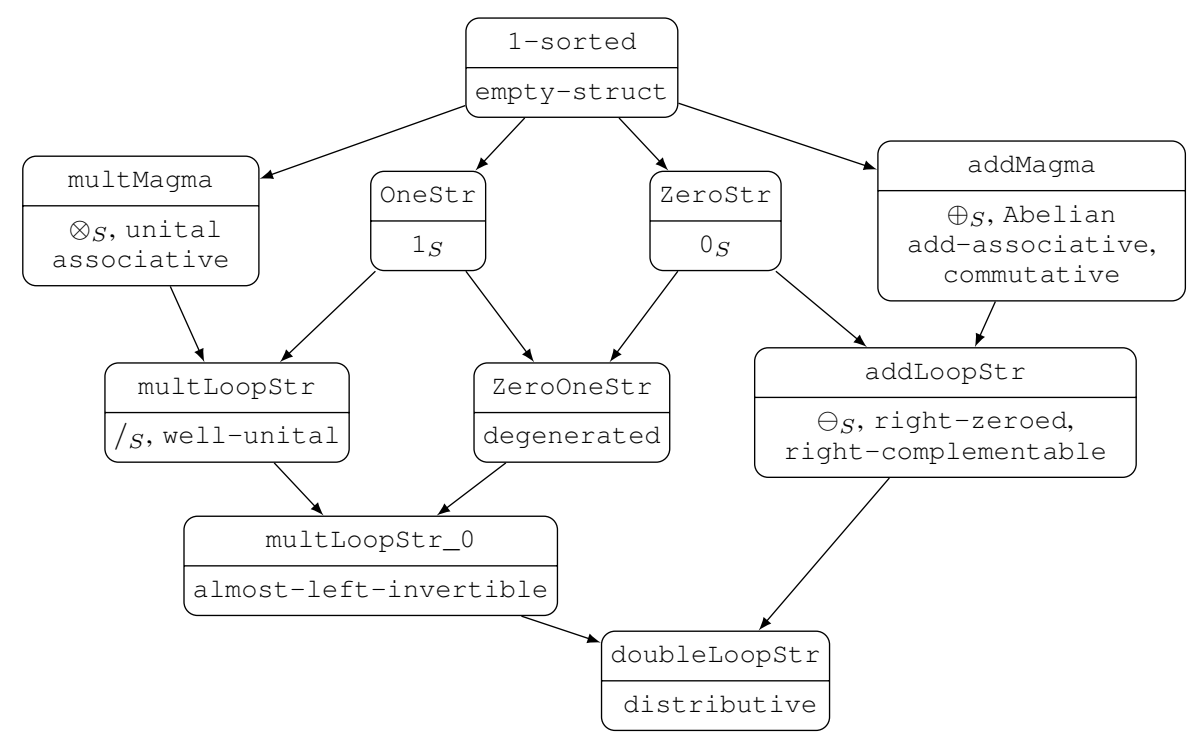

Figure 2. The net of doubleLoopstr structure signature ancestors in the MML. For each node, the adjectives required to define a field as well as the unary and binary operations performed on the elements, are listed below.

The basic structures are depicted in Fig. 1. These 15 signatures are the direct ancestors of 57 other structure signatures in the MML. Furthermore, these 15 are directly used to define 293 Mizar types (this includes non-expandable types [36]), 291 attributes, 962 functors, and 91 predicates.

As structure signatures are mostly used with adjectives, we reformalize the chosen structures along with their attributes to demonstrate that they can be used efficiently in subsequent proofs. In the paper we focus on doubleLoopstr, defined in the MML article ALGSTR_0:

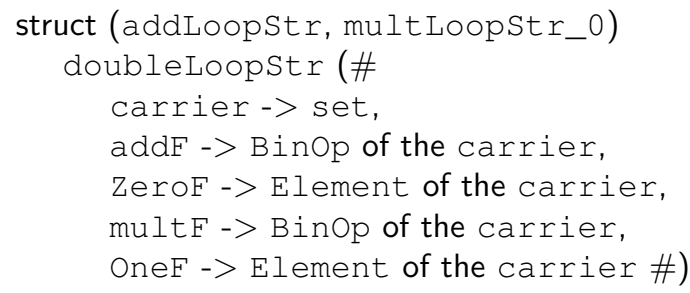

that inherits from both addLoopStr and multLoopstr_0, i.e., the signatures of additive and multiplicative groups, respectively. The doubleLoopstr structure is also the direct ancestor of the signature or ModuleStrover F used in vector space domains, where $F$ represents the set of scalar values. A correct definition of ModuleStrover F permits us to verify our model and our approach for structures parametrized by other structures.

\section{A. Field Formalization}

For our formalization of the signature and basic properties of fields, it was necessary to adapt $20 \mathrm{MML}$ articles. Our reformalization focused on the articles STRUCT_0, GROUP_1, RLVECT_1, ALGSTR_0, VECTSP_1, which define all the ancestors of a field (doubleLoopstr), and the main adjectives used in the field definitions, as well as the basic binary and unary operations. In particular we completely cover ALGSTR_0 in Isabelle/Mizar, which includes 43 functor and predicate definitions (including 13 correctness condition proofs), 72 registrations: non-emptiness of types and relations between groups of adjectives defined on structures, and 6 signatures including

$$
\begin{aligned}
& \text { definition } \\
& \text { struct doubleLoopStr }(\# \\
& \text { carrier } \rightarrow \text { set'; }^{\prime} \\
& \text { addF } \rightarrow \text { BinOp-of' } \text { the }^{\prime} \text { carrier; } \\
& \text { multF } \rightarrow \text { BinOp-of' } \text { the }^{\prime} \text { carrier; } \\
& \text { OneF } \rightarrow \text { Element-of' } \text { the }^{\prime} \text { carrier; } \\
& \text { ZeroF } \rightarrow \text { Element-of } \text { the }^{\prime} \text { carrier \#) }
\end{aligned}
$$

The signature can be used for more complex algebraic structures by extending it by appropriate adjectives. In particular we exactly imitate the MML definitions:

\section{abbreviation \\ Ring $\equiv$ Abelian | add-associative | right-zeroed | right-complementable | associative | well-unital | distributive | non empty-struct $\|$ doubleLoopStr}

\section{abbreviation}

SkewField $\equiv$ non degenerated | almost-left-invertible || Ring

\section{abbreviation}

Field $\equiv$ commutative $\|$ SkewField

The adjectives used in the above definitions have been specified for the various ancestors of doubleLoopstr (see Fig. 2). Such definitions have been moved to earliest possible structures as part of the MML refactoring. This allows easy import of developed theories, which we want to now evaluate in Isabelle/Mizar. Consider the theory of additive groups. It is 

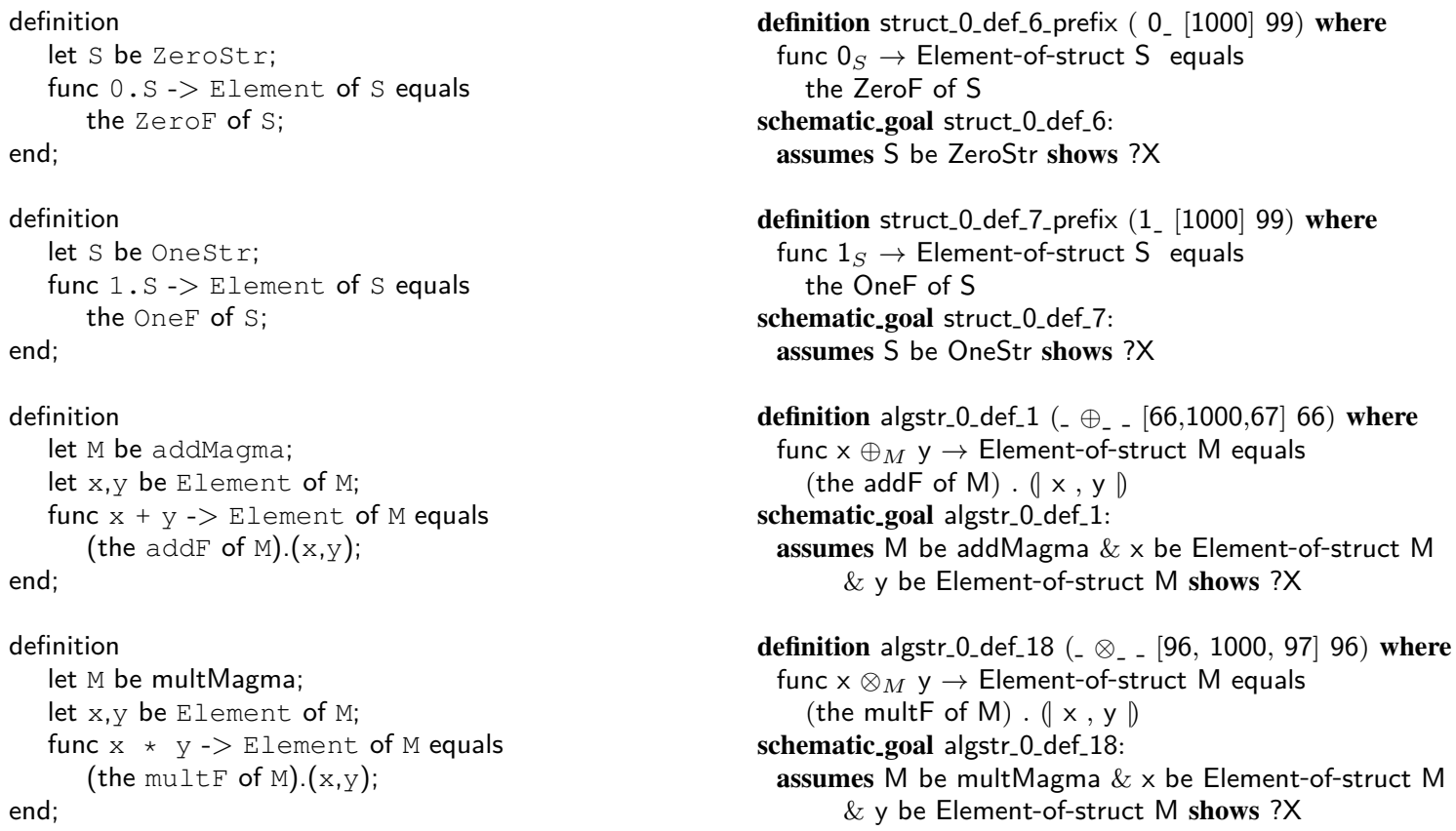

Figure 3. Selected definitions of highlighted elements and binary operations in doubleLoopstr originally formulated in the MML and their Isabelle/Mizar reformulations.
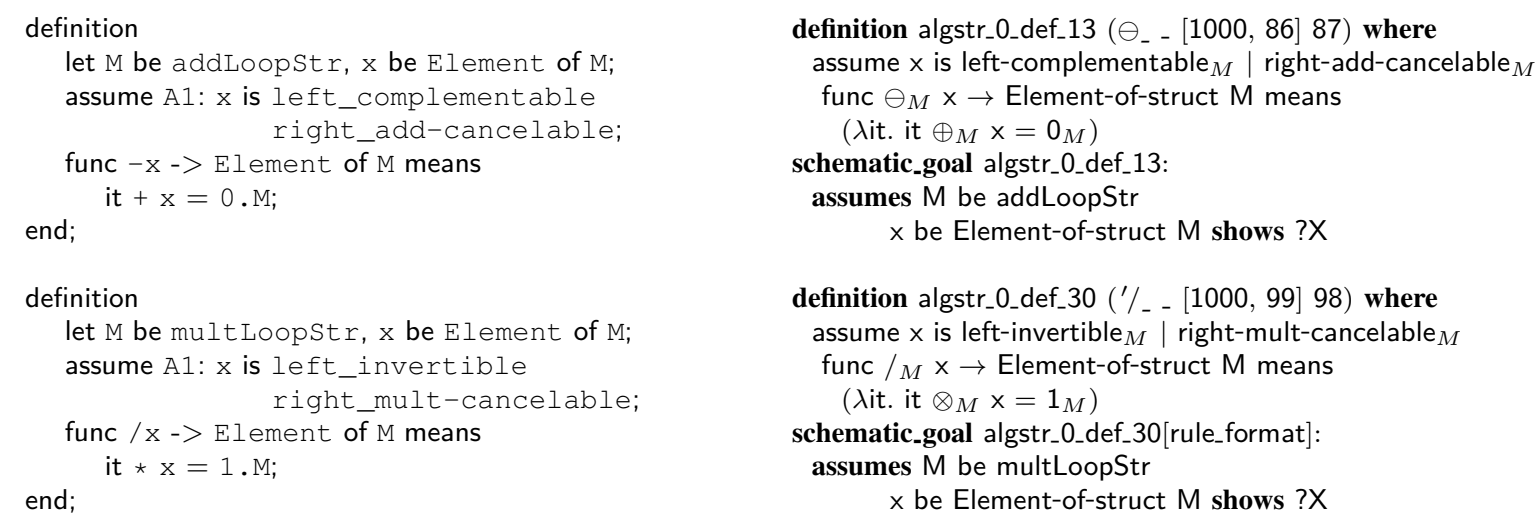

Figure 4. Selected conditional definitions of unary operations from ALGSTR_0 originally formulated in the MML and their Isabelle/Mizar reformulation.

mostly defined over the structure addLoopstr with the adjectives add-associative, right_zeroed right_complementable. As addLoopstr is an ancestor of the doubleLoopstr signature, this set of adjectives is a subset of that used for example for rings, therefore, properties of additive group can be used in the context of rings in the MML.

Moreover, Mizar allows the use of functors defined on ancestors with arguments of further types. We show the definitions of the selected elements and operations of doubleLoopstr, namely $0,1,+$, and $\star$ are defined in Fig. 3. In Mizar, the patterns of the symbols are given in previously specified dictionaries, while in Isabelle these need to be given in the definition block. Furthermore, the definition is split into two parts: the pattern without the argument types and the definition theorems. This allows reducing the number of visible arguments corresponding to hidden arguments in Mizar, as well as allows interpreting conditional definitions (see Fig. 4). The conditions need to appear in the pattern in the Isabelle/Mizar approach. More details are given in the Mizar_defs theory file.

As case studies, we show that the proposed way to model structures and their inheritance is sufficient not only to define attributes and functors but also is adequate for imitating Mizarstyle formalization. For this purpose, we reformalize (so far manually) selected theorems that concern, e.g. the additive and multiplicative groups, and use them in the context of doubleLoopstr. Here we show a single selected proof of the statement that the product of two elements is zero if and only if at least one of them is zero (see Fig. 5). Note that the justification of steps refer to theories developed 


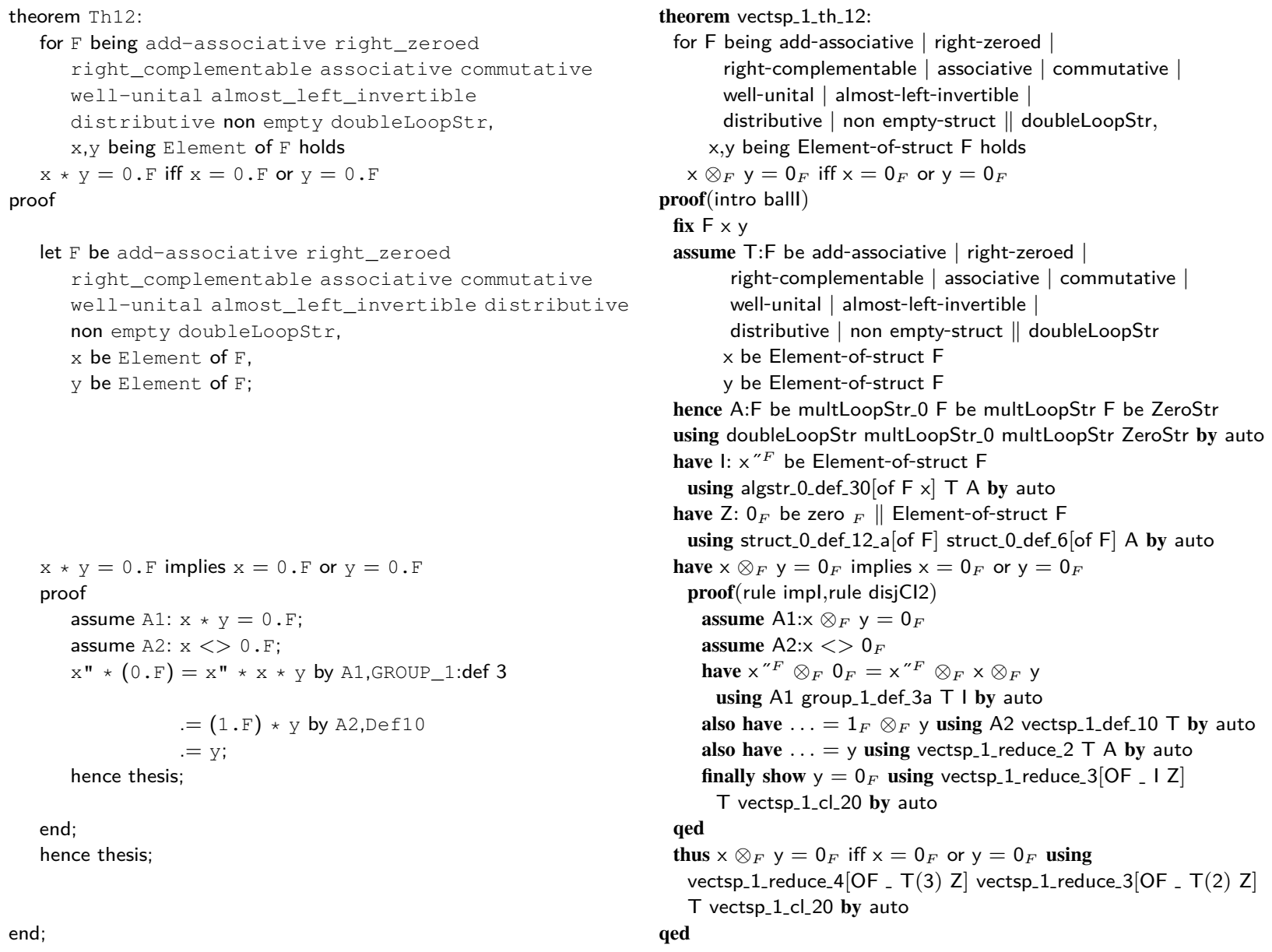

Figure 5. A property of fields originally formulated in the VECTSP_1 article and its Isabelle/Mizar reformulation.

for mult LoopStr_0, multLoopStr, ZeroStr structures (see steps that use label $A$ in justifications). Additionally, each such justification uses some of the attributes indicated in the step labeled by $\mathrm{T}$.

We make use of Isabelle features to model the proofs in such a way that the Isabelle/Mizar language can be as close as possible to the Mizar one. This simplifies the comparison of both proofs. The proofs in our certification contain more steps than the Mizar ones. This is mainly due to the lack of the Mizar automation in our system, e.g., type inference, equational calculus [38], definitional expansions [39]. Additionally, we still have to directly indicate the background information, such as registrations, that are processed automatically by Mizar. We are currently working on mechanisms that would reduce the numbers of additional steps required.

\section{CONCLUSION}

We have presented the progress in our project aiming to independently certify Mizar proofs in the Isabelle logical framework. The proposed recursive approach to structures allows more readable proofs of well-definedness, as well as a more concise way to specify structure inheritance. We verified the provided mechanisms by reformalizing the complete Mizar article defining basic algebraic structures ALGSTR_0, as well as parts of several articles that define and prove properties of structures contain other structures as fields. The experiments confirm that the proposed approach is convenient for proving structure properties.

The Isabelle/Mizar formalization currently includes 31 theorems, 97 registrations including 6 reductions, 86 definitions where 37 of them required Mizar-style justifications and 4 redefinitions concerning MML structures. The total size of the development is $416 \mathrm{kB}$ and 9742 lines of code. It is available at:

$$
\text { http://cl-informatik.uibk.ac.at/cek/fedcsis2017/ }
$$

\section{A. Future Work}

Our certification work has so far focused on the foundations, definitions, and registrations available in the Mizar language. A natural next step would be to allow an implicit use of the background knowledge. Without it, redundant steps in reasoning to represent information computed the Mizar type-inference mechanisms have been necessary so far. Furthermore, we plan to translate the whole MML into the Isabelle/Mizar environment in an automated way and with the help of automatically finding related concepts between 
logics [40], as well as by improving the currently available Isabelle automation for Mizar [41] we hope to cross-verify large parts of the translated MML in Isabelle which is also one of the important steps in the creation of a combined formal library spanning multiple foundations and systems [42].

\section{ACKNOWLEDGEMENT}

This work has been supported by the OeAD Scientific \& Technological Cooperation with Poland grant PL 03/2016.

\section{REFERENCES}

[1] X. Leroy, "Formal verification of a realistic compiler," Commun. ACM vol. 52 , no. 7 , pp. $107-115$, 2009. doi: $10.1145 / 1538788.1538814$

[2] G. Klein, J. Andronick, K. Elphinstone, T. C. Murray, T. Sewell, R. Kolanski, and G. Heiser, "Comprehensive formal verification of an OS microkernel," ACM Trans. Comput. Syst., vol. 32, no. 1, p. 2, 2014. doi: $10.1145 / 2560537$

[3] J. Harrison, "Floating-Point Verification," J. UCS, vol. 13, no. 5, pp. 629-638, 2007. doi: 10.3217/jucs-013-05-0629

[4] T. C. Hales, M. Adams, G. Bauer, D. T. Dang, J. Harrison, T. L. Hoang, C. Kaliszyk, V. Magron, S. McLaughlin, T. T. Nguyen, T. Q. Nguyen, T. Nipkow, S. Obua, J. Pleso, J. Rute, A. Solovyev, A. H. T. Ta, T. N Tran, D. T. Trieu, J. Urban, K. K. Vu, and R. Zumkeller, "A formal proof of the Kepler conjecture," Forum of Mathematics, Pi, vol. 5, 2017. doi 10.1017/fmp.2017.1

[5] G. Bancerek, C. Bylinski, A. Grabowski, A. Korniłowicz, R. Matuszewski, A. Naumowicz, K. Pak, and J. Urban, "Mizar: State-ofthe-art and Beyond," in Intelligent Computer Mathematics - International Conference, CICM 2015, ser. LNCS, M. Kerber, J. Carette, C. Kaliszyk, F. Rabe, and V. Sorge, Eds., vol. 9150. Springer, 2015. doi: 10.1007/978-3-319-20615-8_17 pp. 261-279.

[6] J. Alama, M. Kohlhase, L. Mamane, A. Naumowicz, P. Rudnicki, and J. Urban, "Licensing the Mizar Mathematical Library" in Proc. 10th International Conference on Intelligent Computer Mathematics (CICM 2011), ser. LNCS, J. H. Davenport, W. M. Farmer, J. Urban, and F. Rabe, Eds., vol. 6824. Springer, 2011. doi: 10.1007/978-3-642-22673-1_11 pp. $149-163$

[7] M. Wenzel, L. C. Paulson, and T. Nipkow, "The Isabelle framework," in Theorem Proving in Higher Order Logics, 21 st International Conference, TPHOLs 2008, ser. LNCS, O. A. Mohamed, C. A. Muñoz, and S. Tahar, Eds., vol. 5170. Springer, 2008. doi: 10.1007/978-3-540-71067-7_7 pp. 33-38.

[8] J. C. Blanchette, M. Haslbeck, D. Matichuk, and T. Nipkow, "Mining the Archive of Formal Proofs," in Intelligent Computer Mathematics (CICM 2015), ser. LNCS, M. Kerber, J. Carette, C. Kaliszyk, F. Rabe, and V. Sorge, Eds., vol. 9150. Springer, 2015. doi: 10.1007/978-3-31920615-8_1 pp. 3-17.

[9] Y. Bertot, "A Short Presentation of Coq," in Theorem Proving in Highe Order Logics (TPHOLs 2008), ser. LNCS, O. A. Mohamed, C. A. Muñoz, and S. Tahar, Eds., vol. 5170. Springer, 2008. doi: 10.1007/9783-540-71067-7_3 pp. 12-16.

[10] L. Cruz-Filipe, H. Geuvers, and F. Wiedijk, "C-CoRN, the Constructive Coq Repository at Nijmegen," in Mathematical Knowledge Management (MKM'04), ser. LNCS, A. Asperti, G. Bancerek, and A. Trybulec, Eds., vol. 3119. Springer, 2004. doi: 10.1007/978-3-540-27818-4_7 pp. 88 103.

[11] G. Gonthier and A. Mahboubi, "An introduction to small scale reflection in Coq," J. Formalized Reasoning, vol. 3, no. 2, pp. 95-152, 2010. doi: 10.6092/issn.1972-5787/1979

[12] A. Grabowski and T. Mitsuishi, "Formalizing Lattice-Theoretical Aspects of Rough and Fuzzy Sets," in Rough Sets and Knowledge Technology - 10th International Conference, RSKT 2015, held as part of the International Joint Conference on Rough Sets, IJCRS 2015, Tianjin China, November 20-23, 2015, Proceedings, 2015. doi: 10.1007/978-3319-25754-9_31 pp. 347-356.

[13] L. C. Paulson, "Isabelle: The next 700 theorem provers," in Logic and Computer Science (1990), P. Odifreddi, Ed., 1990, pp. 361-386.

[14] C. Kaliszyk, K. Pakk, and J. Urban, "Towards a Mizar Environment for Isabelle: Foundations and Language," in Proc. 5th Conference on Certified Programs and Proofs (CPP 2016), J. Avigad and A. Chlipala, Eds. ACM, 2016. doi: $10.1145 / 2854065.2854070$ pp. 58-65.
[15] C. Kaliszyk and K. Pąk, "Presentation and Manipulation of Mizar Properties in an Isabelle Object Logic," in Intelligent Computer Mathematics - 10th International Conference, CICM 2017, Edinburgh, UK, July 1721, 2017, Proceedings, ser. LNCS, H. Geuvers, M. England, O. Hasan, F. Rabe, and O. Teschke, Eds., vol. 10383. Springer, 2017. doi 10.1007/978-3-319-62075-6_14 pp. 193-207.

[16] C. Schürmann, "The Twelf Proof Assistant," in Theorem Proving in Higher Order Logics, 22nd International Conference, TPHOLs 2009 ser. LNCS, S. Berghofer, T. Nipkow, C. Urban, and M. Wenzel, Eds., vol. 5674. Springer, 2009. doi: 10.1007/978-3-642-03359-9_7 pp. 7983.

[17] F. Rabe, "A logical framework combining model and proof theory," Mathematical Structures in Computer Science, vol. 23, no. 5, pp. 9451001, 2013. doi: 10.1017/S0960129512000424

[18] M. Wenzel, "Isar - A Generic Interpretative Approach to Readable Formal Proof Documents," in Theorem Proving in Higher Order Logics, 12th International Conference, TPHOLs'99, ser. LNCS, Y. Bertot, G. Dowek, A. Hirschowitz, C. Paulin, and L. Théry, Eds., vol. 1690. Springer, 1999. doi: 10.1007/3-540-48256-3_12 pp. 167-184.

[19] M. Wenzel and F. Wiedijk, "A Comparison of Mizar and Isar," J. Autom. Reasoning, vol. 29, no. 3-4, pp. 389-411, 2002. doi 10.1023/A:1021935419355

[20] K. Pąk, "Readable Formalization of Euler's Partition Theorem in Mizar," in Intelligent Computer Mathematics - International Conference, CICM 2015, Washington, DC, USA, July 13-17, 2015, Proceedings, 2015. doi: 10.1007/978-3-319-20615-8_14 pp. 211-226.

[21] K. Pąk, "Automated Improving of Proof Legibility in the Mizar System," in Intelligent Computer Mathematics - International Conference, CICM 2014, Coimbra, Portugal, July 7-11, 2014. Proceedings, 2014. doi: 10.1007/978-3-319-08434-3_27 pp. 373-387.

[22] K. Pak, "Improving Legibility of Formal Proofs Based on the Close Reference Principle is NP-hard," Journal of Automated Reasoning, vol. 55, no. 3, pp. 295-306, 2015. doi: 10.1007/s10817-015-9337-1

[23] J. Harrison, "A Mizar Mode for HOL" in Theorem Proving in Higher Order Logics: 9th International Conference, TPHOLs'96, ser. LNCS, J. von Wright, J. Grundy, and J. Harrison, Eds., vol. 1125. Springer, 1996. doi: $10.1007 / \mathrm{BFb} 0105406$ pp. 203-220.

[24] C. Kaliszyk and F. Wiedijk, "Merging Procedural and Declarative Proof," in Types for Proofs and Programs, International Conference, TYPES 2008, ser. LNCS, S. Berardi, F. Damiani, and U. de'Liguoro, Eds., vol. 5497. Springer, 2008. doi: 10.1007/978-3-642-02444-3_13 pp. 203219.

[25] D. Syme, "Three Tactic Theorem Proving," in Theorem Proving in Higher Order Logics, 12th International Conference, TPHOLs'99, Nice, France, September, 1999, Proceedings, ser. LNCS, Y. Bertot, G. Dowek, A. Hirschowitz, C. Paulin-Mohring, and L. Théry, Eds., vol. 1690 Springer, 1999. doi: 10.1007/3-540-48256-3_14 pp. 203-220.

[26] F. Wiedijk, "Mizar Light for HOL Light," in Theorem Proving in Higher Order Logics, 14th International Conference, TPHOLs 2001, ser. LNCS, R. J. Boulton and P. B. Jackson, Eds., vol. 2152. Springer, 2001. doi: 10.1007/3-540-44755-5_26. ISBN 3-540-42525-X pp. 378-394.

[27] _ - "A Synthesis of the Procedural and Declarative Styles of Interactive Theorem Proving," Logical Methods in Computer Science, vol. 8 , no. 1, 2012. doi: 10.2168/LMCS-8(1:30)2012

[28] P. Corbineau, "A Declarative Language for the Coq Proof Assistant," in Types for Proofs and Programs, International Conference, TYPES 2007, ser. LNCS, M. Miculan, I. Scagnetto, and F. Honsell, Eds., vol. 4941. Springer, 2007. doi: 10.1007/978-3-540-68103-8_5. ISBN 9783-540-68084-0 pp. 69-84

[29] S. Jaśkowski, "On the rules of suppositions," Studia Logica, vol. 1, 1934

[30] J. Urban, "MPTP 0.2: Design, implementation, and initial experiments," J. Autom. Reasoning, vol. 37, no. 1-2, pp. 21-43, 2006. doi: $10.1007 / \mathrm{s} 10817-006-9032-3$

[31] C. Kaliszyk and J. Urban, "MizAR 40 for Mizar 40," J. Autom Reasoning, vol. 55, no. 3, pp. 245-256, 2015. doi: 10.1007/s10817$015-9330-8$

[32] O. Kunčar, "Reconstruction of the Mizar type system in the HOL Light system," in WDS Proceedings of Contributed Papers: Part I Mathematics and Computer Sciences, J. Pavlu and J. Safrankova, Eds. Matfyzpress, 2010, pp. 7-12

[33] A. Grabowski, A. Korniłowicz, and A. Naumowicz, "Mizar in a Nutshell," J. Formalized Reasoning, vol. 3, no. 2, pp. 153-245, 2010. doi 10.6092/issn. 1972-5787/1980 
[34] M. Iancu, M. Kohlhase, F. Rabe, and J. Urban, "The Mizar Mathematical Library in OMDoc: Translation and applications," J. Autom. Reasoning, vol. 50, no. 2, pp. 191-202, 2013. doi: 10.1007/s10817-012-9271-4

[35] L. C. Paulson, "Set theory for verification: I. From foundations to functions," J. Autom. Reasoning, vol. 11, no. 3, pp. 353-389, 1993. doi: 10.1007/BF00881873

[36] A. Grabowski, A. Korniłowicz, and A. Naumowicz, "Four Decades of Mizar,' Journal of Automated Reasoning, vol. 55, no. 3, pp. 191-198, October 2015. doi: 10.1007/s10817-015-9345-1

[37] A. Grabowski, A. Korniłowicz, and C. Schwarzweller, "On Algebraic Hierarchies in Mathematical Repository of Mizar," in Proc. Federated Conference on Computer Science and Information Systems (FedCSIS 2016), M. Ganzha, L. A. Maciaszek, and M. Paprzycki, Eds., 2016. doi: 10.15439/2016F520 pp. 363-371.

[38] A. Grabowski, A. Korniłowicz, and C. Schwarzweller, "Equality in Computer Proof-Assistants," in Proceedings of the 2015 Federated Conference on Computer Science and Information Systems, ser. Annals of Computer Science and Information Systems, M. Ganzha, L. A. Maciaszek, and M. Paprzycki, Eds., vol. 5. IEEE, 2015. doi:
$10.15439 / 2015$ F229 pp. $45-54$.

[39] A. Korniłowicz, "Definitional expansions in Mizar," Journal of Automated Reasoning, vol. 55, no. 3, pp. 257-268, October 2015. doi: 10.1007/s10817-015-9331-7. [Online]. Available: http: //dx.doi.org/10.1007/s10817-015-9331-7

[40] T. Gauthier and C. Kaliszyk, "Matching concepts across HOL libraries," in Proc. of the 7th Conference on Intelligent Computer Mathematics (CICM'14), ser. LNCS, S. Watt, J. Davenport, A. Sexton, P. Sojka, and J. Urban, Eds., vol. 8543. Springer, 2014. doi: 10.1007/978-3-31908434-3_20 pp. 267-281.

[41] C. Kaliszyk and J. Urban, "Learning-assisted Theorem Proving with Millions of Lemmas," Journal of Symbolic Computation, vol. 69, pp. 109-128, 2015. doi: 10.1016/j.jsc.2014.09.032

[42] P. Corbineau and C. Kaliszyk, "Cooperative Repositories for Formal Proofs," in Towards Mechanized Mathematical Assistants, 14th Symposium, Calculemus 2007, 6th International Conference, MKM 2007, Hagenberg, Austria, June 27-30, 2007, Proceedings, ser. LNCS, M. Kauers, M. Kerber, R. Miner, and W. Windsteiger, Eds., vol. 4573. Springer, 2007. doi: 10.1007/978-3-540-73086-6_19 pp. 221-234. 